K. P MOK

KODAI MATH. J.

I (1978), 411-420

\title{
INFINITESIMAL AUTOMORPHISMS ON THE COTANGENT BUNDLE
}

\author{
BY KAM-PING MOK
}

\section{§1. Introduction.}

Let $M$ be an $n$-dimensional differentiable manifold of class $C^{\infty}, T M$ its tangent bundle and $T^{*} M$ its cotangent bundle. The problem of determining infinitesimal affine transformations and infinitesimal isometries on $T M$ has been considered by several authors, including Yano and Kobayashi [9], Tanno [5, 6], Sato [4], Yanamoto [7] and Mok [1].

Starting from a torsion-free affine connection $\nabla$ on $M$, Patterson and Walker [3] have shown how to construct a pseudo-Riemannian metric $\nabla^{R}$ on $T^{*} M$, which they called the Riemann extension of $\nabla$ to $T^{*} M$. In [10], Yano and Patterson have defined the complete lift $\nabla^{C}$ of $\nabla$ to $T^{*} M$ to be the Riemannian connection of the metric $\nabla^{R}$.

In this paper, we shall use the method of adapted frames to determine the most general infinitesimal affine transformation on $\left(T^{*} M, \nabla^{C}\right)$ and also the most general infinitesimal isometry on $\left(T^{*} M, \nabla^{R}\right)$. It is interesting to note that the structure of these infinitesimal automorphisms on $T^{*} M$ are quite similar to the structure of their counterparts on TM.

\section{§ 2. Preliminaries.}

In this section, we shall summarize all the basic definitions and results that are needed later. Most of them are well-known, and details can be found in Yano [8] and Yano and Patterson [10]. Indices $a, b, c, \cdots ; h, i, \jmath, \cdots$ have range in $\{1, \cdots, n\}$, while indices $A, B, C, \cdots ; \lambda, \mu, \nu, \cdots$ have range in $\{1, \cdots, n$; $n+1, \cdots, 2 n\}$. We put $i=n+\imath$. Summation over repeated indices is always implied.

Coordinate systems in $M$ are denoted by $\left(U, x^{h}\right)$, where $U$ is the coordinate neighbourhood and $x^{h}$ the coordinate functions. Components in $\left(U, x^{h}\right)$ of geometric objects on $M$ will be referred to simply as components. We denote the partial differentiation $\frac{\partial}{\partial x^{h}}$ by $\partial_{h}$.

Let $\nabla$ be an affine connection on $M$ with components $\Gamma_{j i}^{h}$. Its covariant dif-

Received July 30, 1977 
ferentiation will again be denoted by the same symbol $\nabla$. The curvature tensor $R$ of $\nabla$ have components $R_{k j i}{ }^{h}$. We shall assume throughout the paper that $\nabla$ is torsion-free.

A vector field $X$ on $M$ with components $X^{h}$ is an infintesimal affine transformation of $\nabla$ if

$$
\partial_{j} \partial_{\imath} X^{h}+X^{a} \partial_{a} \Gamma_{i i}^{h}-\Gamma_{j i}^{a} \partial_{a} X^{h}+\Gamma_{a i}^{h} \partial_{j} X^{a}+\Gamma_{j a}^{h} \partial_{i} X^{a}=0 .
$$

The left hand side of (2.1) are the components

$$
\mathcal{L}_{X} \Gamma_{j i}^{h}=\nabla_{j} \nabla_{\imath} X^{h}+R_{k j i}{ }^{h} X^{k}
$$

of the Lie derivative $\mathcal{L}_{X} \nabla$ of $\nabla$ with respect to $X$. The Lie derivative $\mathcal{L}_{X} R$ of the curvature tensor $R$ is given by

$$
\mathcal{L}_{X} R_{k \jmath i}{ }^{h}=X^{a} \nabla_{a} R_{k j i}{ }^{h}-R_{k \jmath \imath}{ }^{a} \nabla_{a} X^{h}+R_{a j i}{ }^{h} \nabla_{k} X^{a}+R_{k a \imath}{ }^{h} \nabla_{\jmath} X^{a}+R_{k \jmath a}{ }^{h} \nabla_{\imath} X^{a} .
$$

It is known that

$$
\mathcal{L}_{X} R_{k j i}{ }^{h}=\nabla_{k} \mathcal{L}_{X} \Gamma_{j i}^{h}-\nabla_{\mathcal{J}} \mathcal{L}_{X} \Gamma_{k \imath}^{h}+T_{k \jmath}^{a} \mathcal{L}_{X} \Gamma_{a \imath}^{h} .
$$

Hence, $\mathcal{L}_{X} R=0$ if $X$ is an infinitesimal affine transformation.

Let $g$ be a pseudo-Riemannian metric on $M$ with components $g_{j i}$. As usual, $\left\{\begin{array}{l}h \\ j\end{array}\right\}$ is the Christoffel symbol of $g_{j i}$ and $\left[g^{j i}\right]$ is the inverse of the matrix $\left[g_{j i}\right]$. A vector field $X$ on $M$ with components $X^{h}$ is an infinitesimal isometry on $(M, g)$ if

$$
X^{a} \partial_{a} g_{j i}+g_{a j} \partial_{i} X^{a}+g_{a i} \partial_{\jmath} X^{a}=0 .
$$

The left hand side of (2.5) are the components $\mathcal{L}_{X} g_{j i}$ of the Lie derivative $\mathcal{L}_{X} g$ of $g$ with respect to $X$. In terms of the covariant differentiation in $(M, g)$, we have

$$
\mathcal{L}_{X} g_{j \imath}=g_{a}, \nabla_{\imath} X^{a}+g_{a \imath} \nabla_{\jmath} X^{a}
$$

It is also known that

$$
\mathcal{L}_{X}\left\{\begin{array}{c}
h \\
{ }_{j}
\end{array}\right\}=\frac{1}{2} g^{h a}\left(\nabla_{\jmath} \mathcal{L}_{X} g_{\imath a}+\nabla_{\imath} \mathcal{L}_{X} g_{j a}-\nabla_{a} \mathcal{L}_{X} g_{j i}\right) .
$$

Thus an infinitesimal isometry is an infinitesimal affine transformation with respect to the Riemannian connection of $g$.

We shall be using the following identities for a torsion-free affine connection $\nabla$ on $M$ :

$$
\begin{gathered}
R_{k j i}{ }^{h}+R_{j i k}{ }^{h}+R_{i k j}{ }^{n}=0, \\
\nabla_{k} R_{j i l}{ }^{h}+\nabla_{j} R_{i k l}{ }^{h}+\nabla_{i} R_{k j l}{ }^{h}=0,
\end{gathered}
$$




$$
\nabla_{k} \nabla_{\jmath} X^{h}-\nabla, \nabla_{k} X^{h}=R_{k \jmath a}{ }^{h} X^{a} .
$$

(2.8) and (2.9) are respectively Bianchi's first and second identity, while (2.10) is the Ricci identity for a vector field with components $X^{h}$.

Let $\pi: T^{*} M \rightarrow M$ be the canonical projection of $T^{*} M$ onto $M$. The coordinate system $\left(U, x^{h}\right)$ in $M$ induces in a natural way a coordinate system $\left\{\pi^{-1}(U),\left(x^{h}, p_{h}\right)\right\}$ in $T * M$, which we call the induced coordinate system. We sometimes write $p_{h}$ as $x^{\bar{h}}$ and $\left(x^{h}, p_{h}\right)$ as $\left(x^{A}\right)$. Components in $\left\{\pi^{-1}(U), x^{A}\right\}$ of geometric objects on $T^{*} M$ will be referred to simply as components. We denote $\frac{\partial}{\partial p_{h}}$ and $\frac{\partial}{\partial x^{A}}$ by $\partial_{\bar{h}}, \partial_{A}$ respectively.

Let $B$ be a vector field, $C$ a tensor of type $(1,1)$ and $F$ a 1 -form on $M$, and suppose that their components are $B^{h}, C_{h}^{i}$ and $F_{h}$ respectively. They induce vector fields $B^{C}, \gamma C$ and $F^{V}$ on $T^{*} M$ whose components are

$$
B^{C}:\left[\begin{array}{c}
B^{h} \\
-p_{i} \partial_{h} B^{\imath}
\end{array}\right], \gamma C:\left[\begin{array}{c}
0 \\
p_{\imath} C_{h}^{\imath}
\end{array}\right], F^{V}:\left[\begin{array}{c}
0 \\
F_{h}
\end{array}\right] .
$$

$B^{C}$ is called the complete lift of $B$ while $F^{V}$ the vertical lift of $F$. On the other hand, we can prove that a $(2,0)$ tensor field $A$ on $M$ with components $A^{\text {ha }}$ induces a vector field $A^{*}$ on $T^{*} M$ whose components are

$$
A^{*}:\left[\begin{array}{c}
A^{h a} p_{a} \\
\Gamma_{h b}^{j} A^{b \imath} p_{\jmath} p_{\imath}
\end{array}\right]
$$

For a torsion-free affine connection $\nabla$ on $M$, its Riemann extension $\nabla^{R}$ is a pseudo-Riemannian metric on $T^{*} M$ whose components are

$$
\nabla^{R}:\left[\begin{array}{cc}
-2 p_{h} \Gamma_{j i}^{h} & \delta_{j i} \\
\delta_{j i} & 0
\end{array}\right]
$$

Let $\nabla^{C}$ be the complete lift of $\nabla$, i. e., the Riemannian connection associated with the metric $\nabla^{R}$. It is known that the non-zero components $\tilde{\Gamma}_{C B}^{A}$ of $\nabla^{C}$ are

$$
\begin{aligned}
& \tilde{\Gamma}_{j i}^{h}=\Gamma_{j i}^{h}, \tilde{\Gamma}_{j i}^{\bar{n}}=p_{a}\left(\partial_{h} \Gamma_{j i}^{a}-\partial_{j} \Gamma_{i h}^{a}-\partial_{\imath} \Gamma_{j h}^{a}+2 \Gamma_{h b}^{a} \Gamma_{j i}^{b}\right), \\
& \tilde{\Gamma}_{j i}^{\bar{n}}=-\Gamma_{j h}^{i}, \quad \tilde{\Gamma}_{j i}^{\bar{n}}=-\Gamma_{h i}^{j} .
\end{aligned}
$$

\section{$\S 3$. Adapted frames in $T * M$.}

With the affine connection $\nabla$ given on $M$, we can introduce on each induced coordinate neighbourhood $\pi^{-1}(U)$ of $T^{*} M$ a frame field which is very useful in our computations. It is called the adapted frame on $\pi^{-1}(U)$ and consists of the following $2 n$ linearly independent vector fields $\left\{D_{\lambda}\right\}=\left\{D_{\jmath}, D_{\jmath}^{-}\right\}$on $\pi^{-1}(U)$ :

$$
D_{\jmath}=\frac{\partial}{\partial x^{\jmath}}+p_{h} \Gamma_{j i}^{h} \frac{\partial}{\partial p_{\imath}}, \quad D_{\jmath}=\frac{\partial}{\partial p_{\jmath}} .
$$


In what follows, we shall often consider the components of tensors and affine connections on $T^{*} M$ with respect to the adapted frame on $\pi^{-1}(U)$. We call such components the frame components to distinguish them from the ordinary components of $\S 2$. By using (3.1), it is not difficult to show that the frame components of the vector fields $\gamma C$ and $F^{V}$ are the same as the ordinary components, namely

$$
r C:\left[\begin{array}{c}
0 \\
p_{i} C_{h}^{2}
\end{array}\right], \quad F^{V}:\left[\begin{array}{c}
0 \\
F_{h}
\end{array}\right]
$$

while the frame components of $B^{C}$ and $A^{*}$ are

$$
B^{C}:\left[\begin{array}{c}
B^{h} \\
-p_{\imath} \nabla_{h} B^{\imath}
\end{array}\right], \quad A^{*}:\left[\begin{array}{c}
A^{h a} p_{a} \\
0
\end{array}\right]
$$

Similarly, the frame components of the Riemann extension $\nabla^{R}$ are easily seen to be

$$
\nabla^{R}:\left[G_{\lambda / l}\right]=\left[\begin{array}{ll}
0 & \delta_{j i} \\
\delta_{j i} & 0
\end{array}\right]
$$

Let $\nabla^{C}$ be the complete lift of $\nabla$. It has been shown in Mok [2] that the possibly non-zero frame components $\tilde{\Gamma}_{\lambda \mu}^{\nu}$ of $\nabla^{C}$ are

$$
\tilde{\Gamma}_{\jmath l}^{h}=\Gamma_{j i}^{h}, \quad \tilde{\Gamma}_{j i}^{\bar{h}}=p_{a} R_{h \imath \jmath}{ }^{a}, \quad \tilde{\Gamma}_{j i}^{\bar{h}_{i}}=-\Gamma_{\jmath h}^{\imath},
$$

and that the possibly non-zero frame components $\widetilde{R}_{\omega \lambda \mu^{\nu}}{ }^{\nu}$ of the curvature tensor $\tilde{R}$ of $\nabla^{C}$ are

$$
\begin{aligned}
& \tilde{R}_{k j i}{ }^{h}=R_{k j i}{ }^{h}, \quad \widetilde{R}_{k j i}{ }^{\bar{h}}=p_{a}\left(\nabla_{k} R_{h \imath \jmath}{ }^{a}-\nabla_{j} R_{h i k}{ }^{a}\right), \\
& \tilde{R}_{k j i}{ }^{\bar{h}}=-R_{k j h}{ }^{2}, \quad \widetilde{R}_{k j i}{ }^{\bar{h}}=-\widetilde{R}_{j k \imath}{ }^{\bar{h}}=-R_{h i k}{ }^{j} .
\end{aligned}
$$

If $\tilde{X}$ is a vector field on $T^{*} M$ with frame components $\tilde{X}^{\nu}$, it can be shown that the frame components $\nabla_{\lambda}^{c} \tilde{X}^{\nu}$ of the covariant derivative $\nabla^{C} \tilde{X}$ of $\tilde{X}$ are given by

$$
\nabla_{\lambda}^{c} \tilde{X}^{\nu}=D_{\lambda}\left(\tilde{X}^{\nu}\right)+\tilde{\Gamma}_{\lambda \mu}^{\nu} \tilde{X}^{\mu}
$$

There are formulas analogous to (3.7) for tensor fields of other types.

\section{$\S 4$. Decomposition of infinitesimal affine transformation.}

Suppose $\tilde{X}$ is an infinitesimal affine transformation on $\left(T^{*} M, \nabla^{C}\right)$. By (2.1), its components $\tilde{X}^{A}$ satisfy

$$
\partial_{C} \partial_{B} \tilde{X}^{A}+\tilde{X}^{E} \partial_{E} \tilde{\Gamma}_{C B}^{A}-\tilde{\Gamma}_{C B}^{E} \partial_{E} \tilde{X}^{A}+\tilde{\Gamma}_{E B}^{A} \partial_{C} \tilde{X}^{E}+\tilde{\Gamma}_{C E}^{A} \partial_{B} \tilde{X}^{E}=0
$$

where $\tilde{\Gamma}_{C B}^{A}$ are the components of $\nabla^{C}$ given in (2.14).

Let us put $(A C B)=(h \bar{j} \bar{\imath})$ in (4.1). We get $\partial_{j} \partial_{i} \tilde{X}^{h}=0$ and so 


$$
\tilde{X}^{h}=A^{h a} p_{a}+B^{h}
$$

where $A^{h a}, B^{h}$ are functions of $x^{h}$ only. It is easy to see that $A^{h a}$ and $B^{h}$ are respectively the components of a $(2,0)$ tensor $A$ and a vector field $B$ on $M$.

If we put $(A C B)=(h j \bar{\imath})$ in $(4.1)$ and use (4.2), we get

$$
\partial_{\jmath} A^{h \imath}+\Gamma_{\jmath a}^{i} A^{h a}+\Gamma_{\jmath a}^{h} A^{a \imath}=0,
$$

i. e., $\nabla A=0$.

If we put $(A C B)=(h j i)$ in (4.1) and use (4.2), considering only those terms not involving $p_{h}$, we get

$$
\partial_{j} \partial_{i} B^{h}+B^{b} \partial_{b} \Gamma_{j i}^{h}-\Gamma_{j i}^{b} \partial_{b} B^{h}+\Gamma_{b i}^{h} \partial_{j} B^{b}+\Gamma_{j b}^{h} \partial_{i} B^{b}=0,
$$

which is the condition for $B$ to be an infinitesimal affine transformation.

Let us now put $(A C B)=(\bar{h} \bar{\imath})$ in (4.1) and use (4.2). We get

$$
\partial_{j} \partial_{i} \tilde{X}^{\tilde{h}}=\Gamma_{h b}^{i} A^{b j}+\Gamma_{h b}^{j} A^{b \imath} .
$$

Hence, $\tilde{X}^{\bar{h}}$ can be expressed in the form

$$
\tilde{X}^{\bar{h}}=\Gamma_{h b}^{j} A^{b \imath} p_{j} p_{i}+E_{h}^{i} p_{\imath}+F_{h},
$$

where $E_{h}^{i}, F_{h}$ are functions of $x^{h}$ only.

It now follows that $\left[\begin{array}{c}B^{h} \\ E_{h}^{i} p_{\imath}+F_{h}\end{array}\right]$ are the components of the vector field $\tilde{X}-A^{*}$. By looking at its transformation law, it is easy to see that $F_{h}$ are the components of a 1 -form $F$ on $M$. Thus, $\left[\begin{array}{c}B^{h} \\ E_{h}^{i} p_{2}\end{array}\right]$ are the components of the vector field $\tilde{X}-A^{*}-F^{v}$. Guided by the form of the components of $B^{C}$ (cf. (2.11)), we put $E_{h}^{i}=-\partial_{h} B^{2}+C_{h}^{i}$. Then $\left[\begin{array}{c}0 \\ C_{h}^{i} p_{\imath}\end{array}\right]$ are the components of the vector field $\tilde{X}-A^{*}-F^{v}-B^{C}$. By looking at its transformation law, we see that $C_{h}^{i}$ are the components of a $(1,1)$ tensor $C$ on $M$ and that

$$
\tilde{X}-A^{*}-F^{V}-B^{C}=r C \text {. }
$$

Let us obtain further information on $C$. We put $(A C B)=(\bar{h} j \bar{\imath})$, use $(4.2)$ and (4.5) and consider only those terms not involving $p_{h}$. What we get is

$$
-\mathcal{L}_{B} \Gamma_{j h}^{i}+\nabla_{j} C_{h}^{i}=0
$$

As $B$ is an infinitesimal affine transformation, we see that $\nabla C=0$.

We summarize what we obtain so far in

Proposition 4.1. Let $\nabla^{C}$ be the complete lift of a torsion-free affine connection $\nabla$ on $M$ to $T^{*} M$. An infinitesimal affine transformation $\tilde{X}$ on $\left(T^{*} M, \nabla^{C}\right)$ can be expressed uniquely in the form 


$$
\tilde{X}=A^{*}+B^{C}+\gamma C+F^{v},
$$

where $A$ is a $(2,0)$ tensor field on $M$ satisfying $\nabla A=0$, $B$ is an infinitesimal affine transformation on $(M, \nabla)$, $C$ is a $(1,1)$ tensor field on $M$ satisfying $\nabla C=0$

and $F$ is a 1-form on $M$.

We can obtain further conditions on $A, B, C, F$ by considering terms involving $p_{h}$ after putting $(A C B)=(h j i)$ in $(4.1)$ and so on. In theory, by carrying this process to the end, we should get the general form of an infinitesimal affine transformation on $\left(T^{*} M, \nabla^{C}\right)$. But in practice, this process becomes more and more involved and tends to be unmanageable. However, having succeeded in decomposing an infinitesimal affine transformation as the sum of vector fields, we now switch to the adapted frames.

\section{$\S 5$. Infinitesimal affine transf ormations on $\left(T^{*} M, \nabla^{C}\right)$.}

Let $\tilde{X}$ be an infinitesimal affine transformation on $\left(T^{*} M, \nabla^{C}\right)$ and suppose that it is expressed uniquely in the form $\tilde{X}=A^{*}+B^{C}+\gamma C+F^{v}$ as in Proposition 4.1. By (3.2) and (3.3), the frame components $\tilde{X}^{\nu}$ of $\tilde{X}$ are given by

$$
\left[\tilde{X}^{\nu}\right]=\left[\begin{array}{c}
A^{h a} p_{a}+B^{h} \\
p_{i}\left(C_{h}^{i}-\nabla_{h} B^{i}\right)+F_{h}
\end{array}\right] .
$$

In what follows, we shall compute the frame components $\mathcal{L}_{\tilde{X}} \tilde{\Gamma}_{\lambda \mu}^{\nu}$ of $\mathcal{L}_{\tilde{X}} \nabla^{c}$ according to (cf. (2.2))

$$
\mathcal{L}_{\tilde{X}} \tilde{\Gamma}_{\lambda \mu}^{\nu}=\nabla_{\lambda}^{C} \nabla_{\mu}^{C} \tilde{X}^{\nu}+\tilde{R}_{\omega \lambda \mu}{ }^{\nu} \tilde{X}^{\omega}
$$

By equating $\mathcal{L}_{\tilde{X}} \tilde{\Gamma}_{\lambda \mu}^{\nu}$ to zero, we then get the conditions on $A, B, C, F$ for $\tilde{X}$ to be an infinitesimal affine transformation. We do our computations in stages.

First, the frame components $\nabla_{\mu}^{c} \tilde{X}^{\nu}$ of $\nabla^{C} \tilde{X}$ can be calculated by using (5.1), (3.5) and (3.7). The expressions thus obtained, after simplification by the conditions on $A, B, C$ in Proposition 4.1, become

$$
\begin{aligned}
& \nabla_{\imath}^{C} \tilde{X}^{h}=\nabla_{i} B^{h}, \\
& \nabla_{i}^{C} \tilde{X}^{h}=A^{h \imath}, \\
& \nabla_{\imath}^{c} \tilde{X}^{\bar{n}}=p_{a} p_{c} R_{h b \imath}{ }^{a} A^{b c}+p_{a} R_{h i b}{ }^{a} B^{b}+\nabla_{i} F_{h}, \\
& \nabla_{i}^{C} X^{\bar{h}}=C_{h}^{i}-\nabla_{h} B^{\imath} .
\end{aligned}
$$

We remark that to obtain the coefficient of $p_{a}$ in $\nabla_{l}^{c} \tilde{X}^{\bar{n}}$, we have to use the Ricci identity (2.10) for the vector field $B$.

Next, the frame components of $\nabla_{\lambda}^{C} \nabla_{\mu}^{c} \tilde{X}^{\nu}$ can be calculated by using (5.3) and (3.5). The expressions thus obtained, after simplification by the conditions on $A, B, C$ in Proposition 4.1, become 


$$
\begin{aligned}
& \nabla_{\jmath}^{C} \nabla_{\iota}^{c} \tilde{X}^{h}=\nabla_{\jmath} \nabla_{i} B^{h}-p_{a} R_{b \imath \jmath}{ }^{a} A^{h b}, \\
& \nabla_{j}^{C} \nabla_{i}^{C} \tilde{X}^{h}=\nabla_{j}^{C} \nabla_{i}^{C} \tilde{X}^{h}=\nabla_{j}^{C} \nabla_{i}^{C} \tilde{X}^{h}=0, \\
& \nabla_{j}^{C} \nabla_{\imath}^{C} \tilde{X}^{\tilde{h}}=p_{a} p_{c} \nabla_{j} R_{h b \imath}{ }^{a} A^{b c}+p_{a}\left[\nabla_{j}\left(R_{h i b}{ }^{a} B^{b}\right)+R_{h b j}{ }^{a} \nabla_{i} B^{b}+R_{b \imath \jmath}{ }^{a} \nabla_{h} B^{b}\right. \\
& \left.-R_{b \imath \jmath}{ }^{a} C_{h}^{b}\right]+\nabla, \nabla_{\imath} F_{h}, \\
& \nabla_{j}^{C} \nabla_{i}^{C} \tilde{X}^{\tilde{n}}=-\nabla_{j} \nabla_{h} B^{\imath}+p_{a} R_{h b j}{ }^{a} A^{b \imath}, \\
& \nabla_{j}^{C} \nabla_{\imath}^{C} \tilde{X}^{\bar{h}}=p_{a}\left(R_{h b \imath}{ }^{\jmath} A^{b a}+R_{h b \imath}{ }^{a} A^{b \jmath}\right)+R_{h i b}{ }^{\jmath} B^{b}, \\
& \nabla_{j}^{C} \nabla_{i}^{C} \tilde{X}^{\bar{h}}=0 \text {. }
\end{aligned}
$$

Finally, the frame components of $\mathcal{L}_{\tilde{X}} \tilde{\Gamma}_{\lambda \mu}^{\mu}$ can be calculated by using (5.2), (5.4), (3.6) and (5.1). The expressions thus obtained, after simplifications by the conditions on $A, B, C$ in Proposition 4.1, become

$$
\begin{aligned}
& \mathcal{L}_{\tilde{X}} \tilde{\Gamma}_{j i}^{h}=p_{a}\left(R_{b j i}{ }^{h} A^{b a}-R_{b \imath \jmath}{ }^{a} A^{h b}\right), \\
& \mathcal{L}_{\tilde{X}} \tilde{\Gamma}_{j i}^{h}=\mathcal{L}_{\tilde{X}} \tilde{\Gamma}_{j i}^{h}=\mathcal{L}_{\tilde{X}} \tilde{\Gamma}_{\tilde{j} i}^{h}=0 \text {, } \\
& \mathcal{L}_{\tilde{X}} \tilde{\Gamma}_{j i}^{\bar{h}}=p_{a} p_{c}\left(\nabla_{b} R_{h \imath \jmath}{ }^{a}-\nabla_{j} R_{b i h}{ }^{a}\right) A^{b c} \\
& +p_{a}\left[\mathcal{L}_{B} R_{h \imath \jmath}{ }^{a}+\left(R_{h \imath \jmath}{ }^{b} C_{b}^{a}-R_{b \imath \jmath}{ }^{a} C_{h}^{b}\right)\right]+\nabla_{\jmath} \nabla_{i} F_{h}+R_{h \imath \jmath}{ }^{b} F_{b}, \\
& \mathcal{L}_{\tilde{X}} \tilde{\Gamma}_{j i}^{\bar{n}}=p_{a}\left(R_{h b j}{ }^{a} A^{b \imath}-R_{b j h}{ }^{2} A^{b a}\right), \\
& \mathcal{L}_{\tilde{X}} \tilde{\Gamma}_{\bar{j} i}^{\bar{n}}=p_{a}\left(R_{h b \imath}{ }^{a} A^{b \jmath}-R_{b i h}{ }^{j} A^{b a}\right), \\
& \mathcal{L}_{\tilde{X}} \tilde{I}^{\overline{1}}{ }_{j i}^{\bar{h}}=0 \text {. }
\end{aligned}
$$

Let us analyse the conditions we get when we equate (5.5) to zero. Apart from the expression for $\mathcal{L}_{\tilde{Y}} \tilde{\Gamma}_{j i}^{\bar{h}}$, the rest of (5.5) when equated to zero is equivalent to

$$
R_{b j i}{ }^{h} A^{b a}=R_{i b j}{ }^{a} A^{b h}=R_{b \imath j}{ }^{a} A^{h b} .
$$

Let us assume (5.6) and try to simplify the expression for $\mathcal{L}_{\bar{X}} \tilde{\Gamma}_{j i}^{\bar{n}}$. We first write $p_{a} p_{c}\left(\nabla_{b} R_{h \imath \jmath}{ }^{a}-\nabla_{j} R_{b i h}{ }^{a}\right) A^{b c}$ as

$$
\begin{aligned}
\frac{1}{2} p_{a} p_{c} & {\left[\left(\nabla_{b} R_{h \imath \jmath}{ }^{a}-\nabla_{j} R_{b i h}{ }^{a}\right) A^{b c}+\left(\nabla_{b} R_{h \imath \jmath}{ }^{c}-\nabla_{j} R_{b i h}{ }^{c}\right) A^{b a}\right] } \\
= & -\frac{1}{2} p_{a} p_{c}\left[\left(\nabla_{h} R_{i b \jmath}{ }^{a}+\nabla_{i} R_{b h \jmath}{ }^{a}+\nabla_{j} R_{b i h}{ }^{a}\right) A^{b c}\right. \\
& \left.+\left(\nabla_{h} R_{i b j}{ }^{c}+\nabla_{i} R_{b h \jmath}{ }^{c}+\nabla_{j} R_{b i h}{ }^{c}\right) A^{b a}\right],
\end{aligned}
$$

using the Bianchi's second identity (2.9). We then use (5.6) to transform it into 


$$
\begin{gathered}
-\frac{1}{2} p_{a} p_{c}\left[\left(\nabla_{h} R_{i b j}{ }^{a}+\nabla_{i} R_{b h j}{ }^{a}+\nabla_{j} R_{b i h}{ }^{a}\right) A^{b c}\right. \\
\left.+\left(\nabla_{h} R_{b j i}{ }^{a}+\nabla_{i} R_{j b h}{ }^{a}+\nabla_{j} R_{h b \imath}{ }^{a}\right) A^{b c}\right] \\
=\frac{1}{2} p_{a} p_{c}\left(\nabla_{h} R_{j i b}{ }^{a}+\nabla_{i} R_{h j b}{ }^{a}+\nabla_{j} R_{i h b}{ }^{a}\right) A^{b c} .
\end{gathered}
$$

Thus, $p_{a} p_{c}\left(\nabla_{b} R_{h \imath \jmath}{ }^{a}-\nabla_{j} R_{b i h}{ }^{a}\right) A^{b c}=0$. Next, we note that $\mathcal{L}_{B} R_{h \imath \jmath}{ }^{a}=0$ by the remark following (2.4). Hence, $\mathcal{L}_{\tilde{X}} \tilde{\Gamma}_{j i}^{\bar{n}}$ reduces to

$$
\mathcal{L}_{\tilde{X}} \tilde{\Gamma}_{\jmath \imath}^{\bar{h}}=p_{a}\left(R_{h \imath \jmath}{ }^{b} C_{b}^{a}-R_{b \imath \jmath}{ }^{a} C_{h}^{b}\right)+\nabla, \nabla_{i} F_{h}+R_{h \imath \jmath}{ }^{b} F_{b} .
$$

It follows that the result of equating (5.5) to zero is equivalent to (5.6) and

$$
R_{h \imath \jmath}{ }^{b} C_{b}^{a}=R_{b \imath \jmath}{ }^{a} C_{h}^{b}, \quad \nabla, \nabla_{i} F_{h}+R_{h \imath \jmath}{ }^{b} F_{b}=0 .
$$

Thus, the conditions in Proposition 4.1 together with (5.6) and (5.7) determine the most general infinitesimal affine transformation on $\left(T^{*} M, \nabla^{C}\right)$. We state this as our main result in

THEOREM 5.1. Let $\nabla^{c}$ be the complete lift of a torsion-free affine connection $\nabla$ on $M$ to $T^{*} M$. The most general infinitesimal affine transformation $\tilde{X}$ on $\left(T^{*} M, \nabla^{C}\right)$ can be expressed uniquely in the form

$$
\tilde{X}=A^{*}+B^{C}+\gamma C+F^{v} \text {, }
$$

where $\quad A$ is a $(2,0)$ tensor field on $M$ satisfying

$$
\nabla A=0, \quad R_{b j i}{ }^{h} A^{b a}=R_{i b \jmath}{ }^{a} A^{b h}=R_{b \imath \jmath}{ }^{a} A^{h b},
$$

$B$ is an infinitesimal affine transformation on $(M, \nabla)$,

$C$ is a $(1,1)$ tensor field on $M$ satisfying

$$
\nabla C=0, \quad R_{h \imath \jmath}{ }^{b} C_{b}^{a}=R_{b \imath \jmath}{ }^{a} C_{h}^{b},
$$

and $\quad F$ is a 1 -form satisfying $\nabla_{j} \nabla_{i} F_{h}+R_{h \imath \jmath}{ }^{b} F_{b}=0$.

\section{§ 6. Infinitesimal isometries on $\left(T^{*} M, \nabla^{R}\right)$.}

Let us return to the Riemann extension $\nabla^{R}$ of the affine connection $\nabla$ and determine its infinitesimal isometries. Thus, let $\tilde{X}$ be an infinitesimal isometry on $\left(T^{*} M, \nabla^{R}\right)$. In particular, $\tilde{X}$ is an infinitesimal affine transformation on $\left(T^{*} M\right.$, $\nabla^{C}$ ) (cf. remark following (2.7)) and according to Theorem 5.1, it can be expressed uniquely in the form

$$
\tilde{X}=A^{*}+B^{C}+\gamma C+F^{V}
$$

with $A, B, C, F$ satisfying the conditions listed in Theorem 5.1. What we now do is to compute the frame components $\mathcal{L}_{\hat{X}} G_{\lambda_{\mu}}$ of $\mathcal{L}_{\widetilde{X}} \nabla^{R}$ according to (cf. (2.6)) 


$$
\mathcal{L}_{\widetilde{X}} G_{\lambda \mu}=G_{\lambda \nu} \nabla_{\mu}^{C} \tilde{X}^{\nu}+G_{\mu \nu} \nabla_{\lambda}^{C} \tilde{X}^{\nu}
$$

and then equate the expressions thus obtained to zero.

On substituting the frame components $G_{\lambda \nu}$ and $\nabla_{\mu}^{C} \tilde{X}^{\nu}$ listed in (3.4) and (5.3) into (6.1) and simplifying the resulting expressions by the conditions in Theorem 5.1 , we get the following frame components for $\mathcal{L}_{\widetilde{X}} G_{\lambda_{\ell l}}$ :

$$
\begin{aligned}
& \mathcal{L}_{\widetilde{X}} G_{j i}=p_{a} p_{c}\left(R_{j b \imath}{ }^{a}+R_{i b \jmath}{ }^{a}\right) A^{b c}+\nabla_{j} F_{\imath}+\nabla_{\imath} F_{\jmath}, \\
& \mathcal{L}_{\widetilde{X}} G_{j i}=\mathcal{L}_{\widetilde{X}} G_{i j}=C_{\jmath}^{i}, \\
& \mathcal{L}_{\widetilde{X}} G_{j i}=A^{j i}+A^{\imath \jmath} .
\end{aligned}
$$

We now look at (6.2) in greater detail. First, write $p_{a} p_{c}\left(R_{j b \imath}{ }^{a}+R_{i b}{ }^{a}\right) A^{b c}$ as

$$
\begin{aligned}
& \frac{1}{2} p_{a} p_{c}\left[\left(R_{j b \imath}{ }^{a}+R_{i b \jmath}{ }^{a}\right) A^{b c}+\left(R_{j b \imath}{ }^{c}+R_{i b \jmath}{ }^{c}\right) A^{b a}\right] \\
= & \frac{1}{2} p_{a} p_{c}\left[\left(R_{j b \imath}{ }^{a}+R_{i b \jmath}{ }^{a}\right) A^{b c}+\left(R_{b \imath \jmath}{ }^{a}+R_{b j \imath}{ }^{a}\right) A^{b c}\right] .
\end{aligned}
$$

Thus $p_{a} p_{c}\left(R_{j b \imath}{ }^{a}+R_{i b j}{ }^{a}\right) A^{b c}=0$. Hence, the infinitesimal transformation in Theorem 5.1 is an infinitesimal isometry iff

$$
A^{j i}+A^{\imath \jmath}=0, \quad C_{j}^{i}=0 \text { and } \nabla_{j} F_{\imath}+\nabla_{\imath} F_{j}=0 .
$$

Next, we note that (6.3) implies some of the conditions in Theorem 5.1. In fact, the last equality in

$$
R_{b j i}{ }^{h} A^{b a}=R_{i b j}{ }^{a} A^{b h}=R_{b \imath \jmath}{ }^{a} A^{h b}
$$

is a consequence of $(6.3)_{1}$. On the other hand $(6.3)_{3}$ implies

$$
\nabla, \nabla_{i} F_{h}+R_{h \imath \jmath}{ }^{b} F_{b}=0
$$

because of the following

LEMMA 6.1. Let $\nabla$ be a torsion-free affine connection and $F$ a 1-form on $M$. Let $H_{j i}=\nabla_{j} F_{\imath}+\nabla_{i} F_{\jmath}, F_{\jmath}$ being the components of $F$. Then

$$
\nabla_{\jmath} \nabla_{i} F_{h}+R_{h \imath \jmath}{ }^{b} F_{b}=\frac{1}{2}\left(\nabla_{j} H_{h \imath}+\nabla_{i} H_{h \jmath}-\nabla_{h} H_{j i}\right) .
$$

The proof of Lemma 6.1 is a direct calculation, using the following Ricci identity for a 1 -form on $M$ :

$$
\nabla_{j} \nabla_{h} F_{\imath}-\nabla_{h} \nabla_{j} F_{i}=-R_{j h \imath}{ }^{b} F_{b} .
$$

Finally, we summarize the result of this section in

THEOREM 6.2. Let $\nabla^{R}$ be the Riemann extension of a torsion-free affine connection $\nabla$ on $M$ to $T^{*} M$. The most general infinitesimal isometry $\tilde{X}$ on $\left(T * M, \nabla^{R}\right)$ 
can be expressed unquely in the form

$$
\tilde{X}=A^{*}+B^{C}+F^{V}
$$

where $A$ is a $(2,0)$ tensor field on $M$ satisfying

$$
A^{j i}+A^{\imath \jmath}=0, \quad \nabla A=0, \quad R_{b j i}{ }^{h} A^{b a}=R_{\imath b \jmath}{ }^{a} A^{b h},
$$

$B$ is an infinitesimal affine transformation on $(M, \nabla)$,

and $\quad F$ is a 1 -form on $M$ satisfying $\nabla_{j} F_{\imath}+\nabla_{i} F_{j}=0$.

\section{REFERENCES}

[1] K.P. MoK, Infinitesimal automorphisms on the tangent bundle, J. Math. Kyoio Unıv., 17 (1977), 399-412.

[2] K.P. MoK, Metrics and connections on the cotangent bundle, Kōdai Math. Sem. Rep., 28 (1977), 226-238.

[3.] E. M. Patterson and A.G. Walker, Riemann extensions, Quart. J. Math. Oxford (2), 3 (1952), 19-28.

[4.] K. SATO, Infinitesimal affine transformations of the tangent bundles with Sasaki metric, Tôhoku Math. Journ., 26 (1974), 353-361.

[5] S. TANNo, Infinitesimal isometries on the tangent bundles with complete lift metric, Tensor, 28 (1974), 139-144.

[6] S. TANnO, Killing vectors and geodesic flow vectors on tangent bundles, J. Reine Angew. Math., 282 (1976), 162-171.

[7] H. Yanamoto, The automorphism groups of the tangent bundles equipped with Almost Kählerian structure, Res. Rep. Nagaoka Tech. Coll., 9 (1973), 121-126.

[8] K. YANO, The theory of Lie derivatives and its applications, North-Holland, Amsterdam, 1957.

[9] K. Yano and S. Kobayashi, Prolongations of tensor fields and connections to tangent bundles, II, Journ. Math. Soc. Japan, 18 (1966), 236-246.

[10] K. Yano and E. M. Patterson, Vertical and complete lifts from a manifold to 1ts cotangent bundle, Journ. Math. Soc. Japan, 19 (1967), 91-113.

University OF HONG KONG AND

Hong Kong Polytechnic. 